Published in final edited form as:

Contraception. 2018 March ; 97(3): 264-269. doi:10.1016/j.contraception.2017.10.009.

\title{
Pharmacy access to Ulipristal acetate in major cities throughout the United States $\star$
}

\author{
Maryssa Shigesato a, ${ }^{*}$, Jennifer Eliaa ${ }^{\mathrm{a}}$, Mary Tschann ${ }^{\mathrm{a}}$, Holly Bullock ${ }^{\mathrm{b}}$, Eric Hurwitz ${ }^{\mathrm{c}}$, Yan Yan \\ $\mathbf{W u}^{\mathrm{c}}$, and Jennifer Salcedo ${ }^{\mathrm{a}}$ \\ aDepartment of Obstetrics, Gynecology \& Women's Health, John A. Burns School of Medicine, \\ University of Hawaii at Manoa, Honolulu, $\mathrm{HI}$ \\ bDepartment of Obstetrics \& Gynecology, University of Utah School of Medicine, Salt Lake City, \\ UT
}

'Office of Public Health Studies, University of Hawaii at Manoa, Honolulu, HI

\begin{abstract}
Objective: Ulipristal acetate (UPA) is a prescription emergency contraceptive pill (ECP). Despite the potential for UPA to reduce the risk of unintended pregnancies, a recent study in Hawaii demonstrated less than $3 \%$ of pharmacies stocked UPA and less than $23 \%$ reported the ability to order it. The primary outcome of our study was to assess the availability of UPA in a sample of large cities nationwide.
\end{abstract}

Study design: We conducted a telephone-based secret shopper study of 533 retail pharmacies sampled proportionally from 10 large cities in five geographic regions across the US. Callers represented themselves as uninsured 18-year-old women attempting to fill prescriptions for UPA between February and May 2016. Using a semi-structured questionnaire, callers inquired regarding availability and use of UPA.

Results: Less than $10 \%$ (33/344; 95\% CI: $6.5-12.7 \%)$ of pharmacies indicated the ability to immediately fill a UPA prescription, while $72 \%$ (224/311; 95\% CI: $65.0-77.0 \%)$ of pharmacies without immediate availability reported the ability to order UPA, with the median predicted wait time of $24 \mathrm{~h}$ (IQR: 21.5 to $26.0 \mathrm{~h}$ ).

Conclusion: Despite evidence for increased efficacy of UPA over levonorgestrel (LNG) ECPs, the availability of UPA in a sample of US major cities is extremely limited. Given that ECPs should be taken as soon as possible after unprotected sex, the long wait times when ordering UPA present an access barrier. Efforts to improve the availability of UPA are important to optimize the potential of ECPs to decrease unintended pregnancy following unprotected sex.

Implications: Interventions are needed to address barriers to obtaining UPA from retail pharmacies nationwide.

\footnotetext{
${ }^{\sqrt{3}}$ Conflicts of Interest: None

"Corresponding author. Tel.: +1 808286 2867. maryssas@ hawaii.edu (M. Shigesato).
} 


\section{Keywords}

Emergency contraception; Ulipristal acetate; Pharmacy availability; Contraceptive access

\section{Introduction}

Emergency contraception is used after unprotected sex, when a contraceptive method has been used incorrectly, or after sexual assault to reduce the risk of pregnancy. In 2010, a single $30 \mathrm{mg}$ dose of ulipristal acetate (UPA) was approved as an emergency contraceptive pill (ECP) for use up to $120 \mathrm{~h}$ after unprotected sex. In contrast, levonorgestrel (LNG) is a single $1.5 \mathrm{mg}$ dose ECP approved for use within $72 \mathrm{~h}$ after unprotected sex that maintains some efficacy to $120 \mathrm{~h} \mathrm{[1,2].} \mathrm{As} \mathrm{an} \mathrm{emergency} \mathrm{contraceptive,} \mathrm{LNG} \mathrm{delays} \mathrm{ovulation} \mathrm{but} \mathrm{has}$ no effect once the level of luteinizing hormone (LH) begins to rise, triggering ovulation [1]. However, UPA remains effective in postponing ovulation if given prior to the LH peak [1]. When a decision-analytic model was used to compare the cost-effectiveness of UPA and LNG, it was found that if UPA was used for the 4,176,572 estimated annual ECP uses in the United States (US), it would result in 37,589 fewer unintended pregnancies and save $\$ 116.3$ million annually in healthcare costs compared to LNG ECPs [3]. In addition, UPA may be more effective than LNG at preventing pregnancy in obese and overweight women [4]. Although UPA is a more effective ECP, a recent survey of pharmacy availability of UPA in Hawaii showed that only $2.6 \%$ of retail pharmacies had UPA immediately available (compared to $82.4 \%$ for $\mathrm{LNG}$ ), and only $22.8 \%$ reported the ability to order the medication [5]. To assess if this limited availability exists more widely, we designed a nationwide telephone secret shopper study of US retail pharmacies in major cities. The purpose of this study was to estimate the immediate pharmacy availability of UPA in a sample of cities with populations over 500,000 and the ability of such pharmacies to order UPA on customer request. Secondary analyses included the estimated time to UPA availability when ordered, the availability of ECPs other than UPA, and accuracy of information provided on ECP use by pharmacy staff.

\section{Materials and methods}

\subsection{Overview}

A telephone-based secret shopper study of pharmacies in cities across the US with populations of at least 500,000 people was conducted. We selected 10 such cities representative of different geographic regions of the country: the West (Denver and Seattle), the Midwest (Detroit and Chicago), the Northeast (Philadelphia and Boston), the South (Jacksonville and Charlotte), and Southwest (Los Angeles and Dallas).

\subsection{Sampling}

Our study was powered to estimate the percentage of pharmacies with UPA immediately available within $4 \%$ of the true prevalence (true prevalence $+/-2 \%$ ) with $95 \%$ confidence. We estimated a baseline prevalence of immediate availability of UPA of $5 \%$ and predicted that $5 \%$ of pharmacies sampled would be excluded from analysis. Based on these estimates, we planned to sample 505 randomly selected pharmacies, which was inflated to 535 to 
account for exclusions. The number of pharmacies selected from each city was proportionate to the total number of pharmacies identified in each city. Cities with populations over 500,000 in the 2010 US Census were considered. We randomly selected two cities per region (West, Midwest, South, Northeast, and Southwest) out of the 33 total US cities meeting population criteria. Pharmacies in each city were identified through two online phone books and cross-referenced with chain pharmacy websites, in an attempt to replicate the anticipated actions of consumers. Computerized random stratified sampling was then performed by the statistical consultant and the list of selected pharmacies returned to the study team.

Calls were made from February through May 2016. Pharmacies were defined as independent pharmacies if they had fewer than 5 locations within the city, with the remaining being categorized as chain pharmacies. Pharmacies were excluded if they were not available to the general public (e.g., a pharmacy in a skilled nursing facility), would not accept the study call, could not be reached after three attempts, had an incorrect or disconnected phone number, or were unable to provide information in English.

Callers represented themselves as uninsured 18-year-old women attempting to fill a prescription for ella ${ }^{\circledR}$ (UPA) and followed a semi-structured questionnaire (Fig. 1). Calls were made on weekdays between 8:00 a.m. and 5:00 p.m. in the time zone of the pharmacy location. Callers directed their questions to the pharmacy staff member who answered the phone and did not specifically ask to speak to a pharmacist. Information on immediate availability of UPA and LNG ECPs, ability to order UPA if not immediately available, outof-pocket prices for ECPs, instructions provided on ECP use, and pharmacy and staff details were recorded. The data were then entered and managed using REDCap (Research Electronic Data Capture) electronic data capture tools hosted at University of Hawaii John

A. Burns School of Medicine. Data were analyzed using R statistical software version 3.2.3 (2015). This study was deemed not to be human subjects research by the University of Hawaii Institutional Review Board.

\subsection{Outcome measures}

The primary study aim was to evaluate the immediate availability of UPA. Secondary outcomes included: ability to order UPA and anticipated time to availability, availability of other ECP products, associations of pharmacy type (chain or independent) and region with ECP availability, accuracy of instructions provided on ECP use, and differences in the accuracy of information provided by pharmacists and other pharmacy staff members. Questions assessing the accuracy of ECP information provided included how soon after unprotected sex UPA needs to be taken and differences between UPA and LNG ECPs. Answers for the following questions were recorded: open-ended responses to differences between UPA and LNG (if a difference was indicated; Table 4), interval after unprotected intercourse during which UPA needs to be taken, and names of other ECP options available at the time of the call. Information on call duration and number of call attempts was also gathered. Call times were recorded for all pharmacies included in the analyses. Call times included hold times if the caller was transferred to a different member of the pharmacy staff or placed on hold by the pharmacy for any other reason. 


\subsection{Statistical analysis}

Frequency measures and proportions for the following outcomes were gathered: ability to immediately fill UPA, type of staff member serving as the primary pharmacy contact, ability to order UPA, availability of other ECPs, and number of pharmacies providing correct responses to inquiries regarding differences between UPA and LNG ECPs.

Sample means with 95\% confidence intervals (CIs) were estimated for the following outcomes: call duration, number of call attempts, interval to UPA availability, and ECP cost. For data not normally distributed, medians and interquartile intervals were calculated.

chi-Square and Fisher's exact tests were used to test for differences in the ability to immediately fill a UPA prescription, to order the medication, or on the availability of other ECPs by geographic region and pharmacy type, to compare the accuracy of responses to questions about UPA, and to test for differences by geographic region and pharmacy type in the immediate availability of alternative ECPs. $t$ Tests and ANOVA were used to compare UPA availability between geographic regions, time to anticipated UPA availability, and UPA cost. Outcomes were determined to be statistically significant at an alpha level of .05.

\section{Results}

\subsection{Immediate availability of ulipristal acetate}

Of the 533 pharmacies called, 344 (65\%) met inclusion criteria (Fig. 2). Of pharmacies meeting inclusion criteria, $10 \%$ (33/344) reported immediate availability of UPA (95\% CI: 7-13\%). We found similar results for immediate availability of UPA by region (Table $1 ; \mathrm{p}=$. 15). However, there was a difference in immediate UPA availability among individual cities (Table 2; $\mathrm{p}=.04)$. Of the pharmacies sampled, $12 \%$ (28/233) of chain pharmacies and 5\% $(5 / 111)$ of independent pharmacies had UPA immediately available $(\mathrm{p}=.04)$.

\subsection{Secondary outcome measures}

The median call duration was $4 \min 55 \mathrm{~s}$ (IQR: $3 \min 12 \mathrm{~s}$ to 6 min $51 \mathrm{~s}$ ). The median number of call attempts to reach a pharmacy staff member was one (IQR: 1 ). Secret shoppers spoke primarily with a pharmacist in 97 (28\%) calls and with a non-pharmacist staff member in $92(27 \%)$ calls. The role of the primary pharmacy contact was uncertain in the remaining $155(45 \%)$ calls.

Of the 311 pharmacies that did not have UPA immediately available, $72 \%(224 / 311)$ indicated the ability to order the medication. A difference in the ability to order UPA by region was noted (Table 3; $\mathrm{p}=.01$ ). Additionally, 81\% (167/205) of chain pharmacies reported the ability to order UPA, compared to 55\% (57/106) of independent pharmacies ( $\mathrm{p}<.001)$. Of the 224 pharmacies that reported an ability to order UPA, $221(99 \%)$ were able to provide an estimate of time to medication availability, with the median time reported as 24 h (IQR: 21.5-26.0 h). Pharmacies in different regions reported similar times to UPA availability $(\mathrm{p}=.38)$. There were also similar times to UPA availability among chain and independent pharmacies $(\mathrm{p}=.09)$. 
When asked if there is a difference between UPA and LNG ECPs, 63\% (211/336) of pharmacy respondents indicated a difference, while 25\% (86/336) stated no difference, and $12 \%$ (39/336) indicated uncertainty. When comparing responses based on the role of the primary pharmacy contact person, $82 \%$ (80/97) of callers who spoke to a pharmacist, $49 \%$ (45/92) of callers who spoke to another pharmacy staff person, and 55\% (86/155) of callers who were unable to identify their primary pharmacy contact, were informed of a difference between the medications $(\mathrm{p}=.001$ ). Responses provided regarding differences between UPA and LNG ECPs are seen in Table 4. Of the 211 pharmacies that provided examples of differences between UPA and LNG, 80\% (168/211) were able to provide correct information on at least one difference between UPA and LNG ECPs. Only 22\% (46/211) of pharmacies discussed more than one difference between UPA and LNG.

When asked how long after unprotected sex UPA may be taken, 51\% (114/221) of pharmacies sampled provided the answer of $120 \mathrm{~h}$ (5 days), while 48\% (106/221) of pharmacies reported that UPA must be taken sooner than $120 \mathrm{~h}$ after sex, and less than $1 \%$ $(1 / 211)$ of pharmacies stated that it may be taken more than $120 \mathrm{~h}$ after sex. The accuracy of responses was similar by region ( $\mathrm{p}=.15$ ). Of the 231 pharmacies that provided a response, 30 (14\%) stated that UPA should be taken as soon as possible after unprotected intercourse.

Of the 277 pharmacies able to fill or order UPA, the median quoted out-of-pocket cost was \$50 (IQR: \$47-\$53) and ranged from \$2.59 to \$1200.99, with similar cost among regions $(\mathrm{p}=.86)$. There were also similar out-of-pocket-costs between chain and independent pharmacies $(\mathrm{p}=.5)$.

When asked if the pharmacy had ECPs other than UPA immediately available, $81 \%$ (273/335) of pharmacies responded positively. There were similar results regarding availability of other ECPs among the different geographic regions $(\mathrm{p}=.31)$. However, of the pharmacies sampled, 93\% (211/228) of chain pharmacies and 58\% (62/106) of independent pharmacies indicated they had other ECP formulations available ( $\mathrm{p}<.001)$. Of the 273 pharmacies indicating availability of another ECP option, $99 \%$ of pharmacies indicated availability of $\mathrm{PlanB}^{\circledR}$ (LNG).

\section{Conclusion}

Our study found immediate availability of UPA to be just under $10 \%$ in a sample of US pharmacies in cities with populations over 500,000. Limited information has been published about the availability of UPA in US retail pharmacies, with small local studies reporting availability between $2.6 \%$ in the State of Hawaii and 7\% in Hampshire and Hampden counties in Massachusetts [5,6]. While the immediate availability of UPA across the large US cities appears to be quite limited, our study found that $72 \%$ of pharmacies sampled without immediate availability stated that UPA could be ordered, indicating willingness to dispense the medication. However, the median anticipated delay to receipt of the medication of $24 \mathrm{~h}$ may significantly limit use of this time-sensitive medication [7]. Additionally, it is concerning that over one-quarter of pharmacies sampled reported an inability to order UPA, which presents a significant access barrier. 
Less than $65 \%$ of pharmacies were able to correctly indicate a difference between UPA and LNG and only half of the pharmacies surveyed were able to identify that UPA can be taken up to $120 \mathrm{~h}$ after unprotected sex. In addition, only 4 responses mentioned UPA is more effective than LNG ECPs for overweight women. This inability of pharmacies to provide accurate information on UPA may further impede women's ability to appropriately use the medication when indicated. Although pharmacists were able to provide correct information more often than non-pharmacists, patient callers may be unlikely to ask to speak specifically to a pharmacist, or may not have the opportunity to do so during a call, which may limit the quality of information received.

\subsection{Strengths and limitations}

Strengths of our study included the secret shopper methodology, which provided data that best approximates real-world patient experiences. Additionally, having callers speak to the pharmacy staff person answering the call, rather than specifically asking to speak to the pharmacist, most closely replicates the experience of a patient caller.

A limitation of our study is that our estimate of UPA immediate availability is less precise than our power analysis predicted due to more pharmacies meeting exclusion criteria than anticipated. Future telephone studies querying pharmacies should anticipate a higher percentage of excluded pharmacies, in the range of approximately $35 \%$. Also, while our study was conducted by sampling pharmacies in 10 large and geographically diverse US cities, results may not be representative of all similarly sized cities nationally, nor representative of all areas of the country. It is also possible that UPA access may be even more limited at pharmacies in smaller cities and rural areas (compared to the large cities we sampled), which should be investigated in future studies. Additionally, it is possible that pharmacies unable to be reached may differ systematically from pharmacies in our study regarding immediate UPA availability and other characteristics. However, it is more likely that such pharmacies would be equally inaccessible to patient callers.

\subsection{Implications}

Given the potential for increased clinical and cost effectiveness of UPA over LNG ECPs, the limited availability of UPA in the large US cities we sampled and the long wait times when ordering the medication are concerning. UPA may be even more difficult to access in retail pharmacies of smaller US cities, which should be evaluated in future studies. Clinicians should be strongly encouraged to provide patients with advance prescriptions for UPA and urge women to fill them immediately to increase the likelihood of availability when needed. Also, women should be educated on additional routes of accessing UPA, such as through online pharmacies [8]. Lastly, increased education of clinicians and pharmacy staff is needed to ensure patients are given accurate information and access to this method of reducing unintended pregnancy.

\section{Acknowledgments}

Statistical support for this project was funded through NIH infrastructural grants U54MD007584 and G12MD007601 and the Department of Obstetrics, Gynecology \& Women's Health of the University of Hawaii John A. Burns School of Medicine. The authors sincerely thank the following individuals for their assistance with 
data collection: Naida Besic, Stephanie Cacal, Kristen Lee, Sarah Steele, and An Yang, as well as Dr. Hyeong Jun Ahn who provided statistical consultation.

\section{References}

[1]. Lalitkumar PGL, Berger C, Gemzell-Danielsson K. Emergency contraception. Best Pract Res Clin Endocrinol Metab 2013;27:91-101. [PubMed: 23384749]

[2]. von Hertzen H, Van Look P. Methods of emergency contraception. Fam Plann Perspect 1996;28:52-88. [PubMed: 8777939]

[3]. Bayer LL, Edelman AB, Caughey CB, Rodriguez MI. The price of emergency contraception in the United States: what is the cost-effectiveness of ulipristal acetate versus single-dose levonorgestrel? Contraception 2013;87:385-90. [PubMed: 23040122]

[4]. Glasier A, Cameron ST, Blithe D, Scherrer B, Mathe H, Levy D, et al. Can we identify women at risk of pregnancy despite using emergency contraception? Data from randomized trials of ulipristal acetate and levonorgestrel. Contraception 2011;84:363-7. [PubMed: 21920190]

[5]. Bullock H, Steele S, Kurata N, Tschann M, Kaneshiro B, Salcedo J. Pharmacy access to ulipristal acetate in Hawaii: is a prescription enough? Contraception 2016;93:452-4. [PubMed: 26689477]

[6]. Brant A, White K, St. Marie P. Pharmacy availability of ulipristal acetate emergency contraception: an audit study. Contraception 2014;90(3):338-9.

[7]. Richardson AR, Maltz FN. Ulipristal acetate: review of the efficacy and safety of a newly approved agent for emergency contraception. Clin Ther 2012;34(1):24-36. [PubMed: 22154199]

[8]. Smith N, Cleland K, Wagner B, Trussell J. “I don't know what I would have done.” Women's experiences acquiring ulipristal acetate emergency contraception online from 2011 to 2015. Contraception 2017;95(4):414-8. [PubMed: 27769767] 
Secret Shopper Patient Questionnaire

1. Hi, my doctor gave me a prescription for Ella. If I bring it in to your pharmacy, could I get it filled today? $\square$ Yes $\square$ No

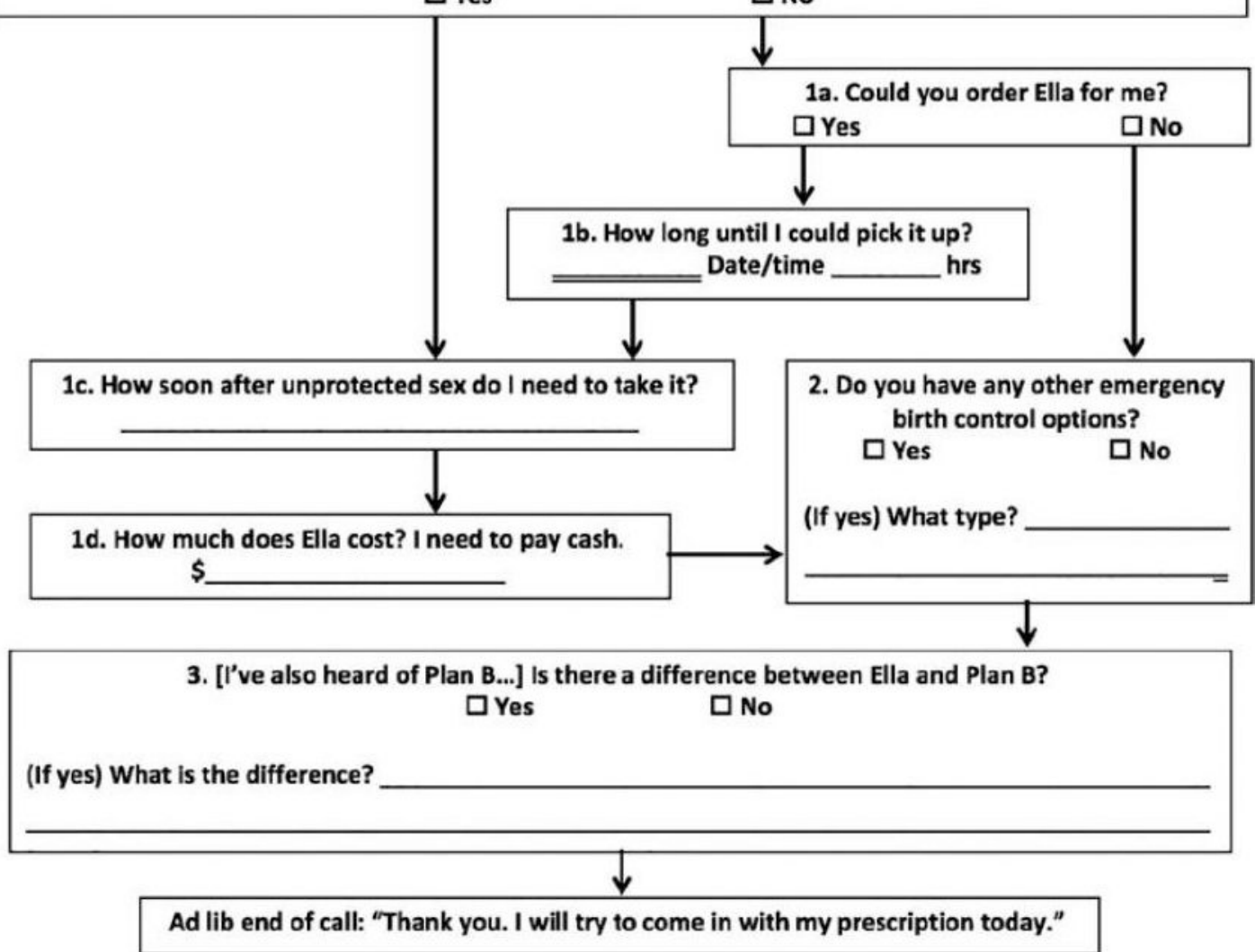

Fig. 1.

Semi-Structured Secret Shopper Questionnaire. 
Total number of pharmacies called $(\mathrm{n}=533)$

- West $=62$

- South $=88$

- Midwest $=137$

- Northeast $=121$

- Southwest $=125$

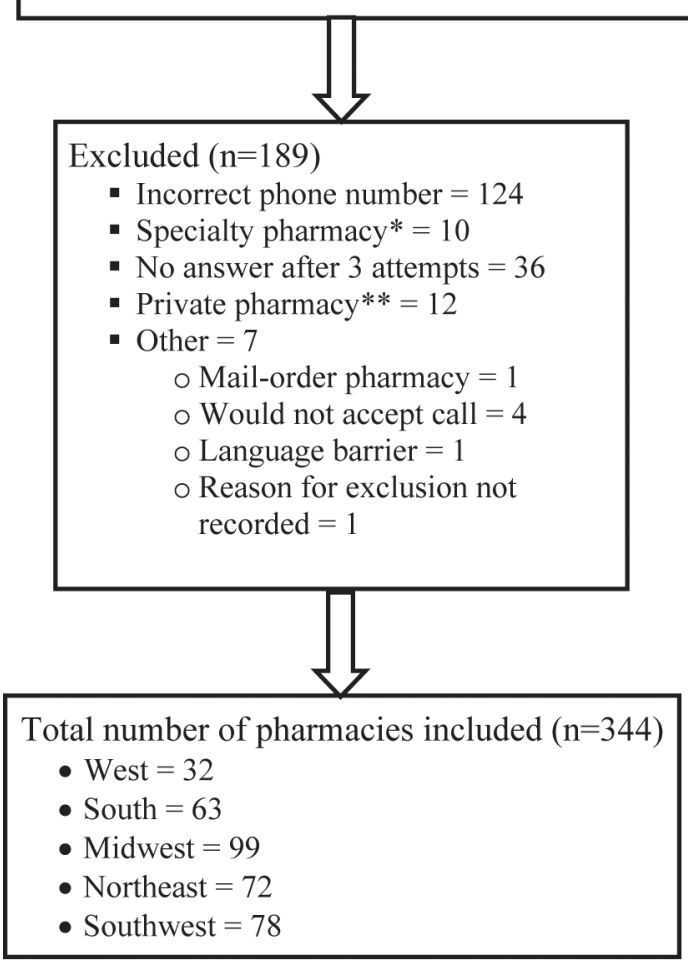

Fig. 2.

Pharmacy Inclusion Criteria. * Specialty pharmacies include pharmacies intended to serve patients with rare, chronic, and/or complex diseases who require specialty medications (e.g., injectable biologic products) and related services. ${ }^{*}$ Private pharmacies include pharmacies not available to the general public, such as those that only service nursing homes. 


\section{Table 1}

Pharmacy ability to immediately fill a UPA prescription, stratified by region.

\begin{tabular}{llcl}
\hline & Total & Yes & No \\
\hline West & $32(9 \%)$ & $7(22 \%)$ & $25(78 \%)$ \\
Midwest & $99(29 \%)$ & $9(9 \%)$ & $90(91 \%)$ \\
Northeast & $72(21 \%)$ & $6(8 \%)$ & $66(92 \%)$ \\
South & $63(18 \%)$ & $3(5 \%)$ & $60(95 \%)$ \\
Southwest & $78(23 \%)$ & $8(10 \%)$ & $70(90 \%)$ \\
Total & 344 & $33(10 \%)$ & $311(90 \%)$ \\
\hline
\end{tabular}

$\mathrm{p}=.15$. 


\section{Table 2}

Pharmacy ability to immediately fill a UPA prescription stratified by city.

\begin{tabular}{lll}
\hline City & Yes & No \\
\hline Boston & $4(17 \%)$ & $20(83 \%)$ \\
Charlotte & $0(0 \%)$ & $26(100 \%)$ \\
Chicago & $9(13 \%)$ & $58(87 \%)$ \\
Dallas & $1(4 \%)$ & $23(96 \%)$ \\
Denver & $4(22 \%)$ & $14(78 \%)$ \\
Detroit & $0(0 \%)$ & $23(100 \%)$ \\
Jacksonville & $3(8 \%)$ & $34(92 \%)$ \\
Los Angeles & $7(13 \%)$ & $47(87 \%)$ \\
Philadelphia & $2(4 \%)$ & $46(96 \%)$ \\
Seattle & $3(21 \%)$ & $11(79 \%)$ \\
\hline
\end{tabular}

$\mathrm{p}=.04$. 


\section{Table 3}

Pharmacy ability to order UPA when not immediately available stratified by region.

\begin{tabular}{llll}
\hline & Total & Yes & \multicolumn{1}{l}{ No } \\
\hline West & $25(8 \%)$ & $21(84 \%)$ & $4(16 \%)$ \\
Midwest & $90(29 \%)$ & $68(76 \%)$ & $22(24 \%)$ \\
Northeast & $66(21 \%)$ & $44(67 \%)$ & $22(33 \%)$ \\
South & $60(19 \%)$ & $50(83 \%)$ & $10(17 \%)$ \\
Southwest & $70(23 \%)$ & $41(59 \%)$ & $29(41 \%)$ \\
Total & 311 & $224(72 \%)$ & $87(28.0 \%)$ \\
\hline
\end{tabular}

$\mathrm{p}=.01$. 


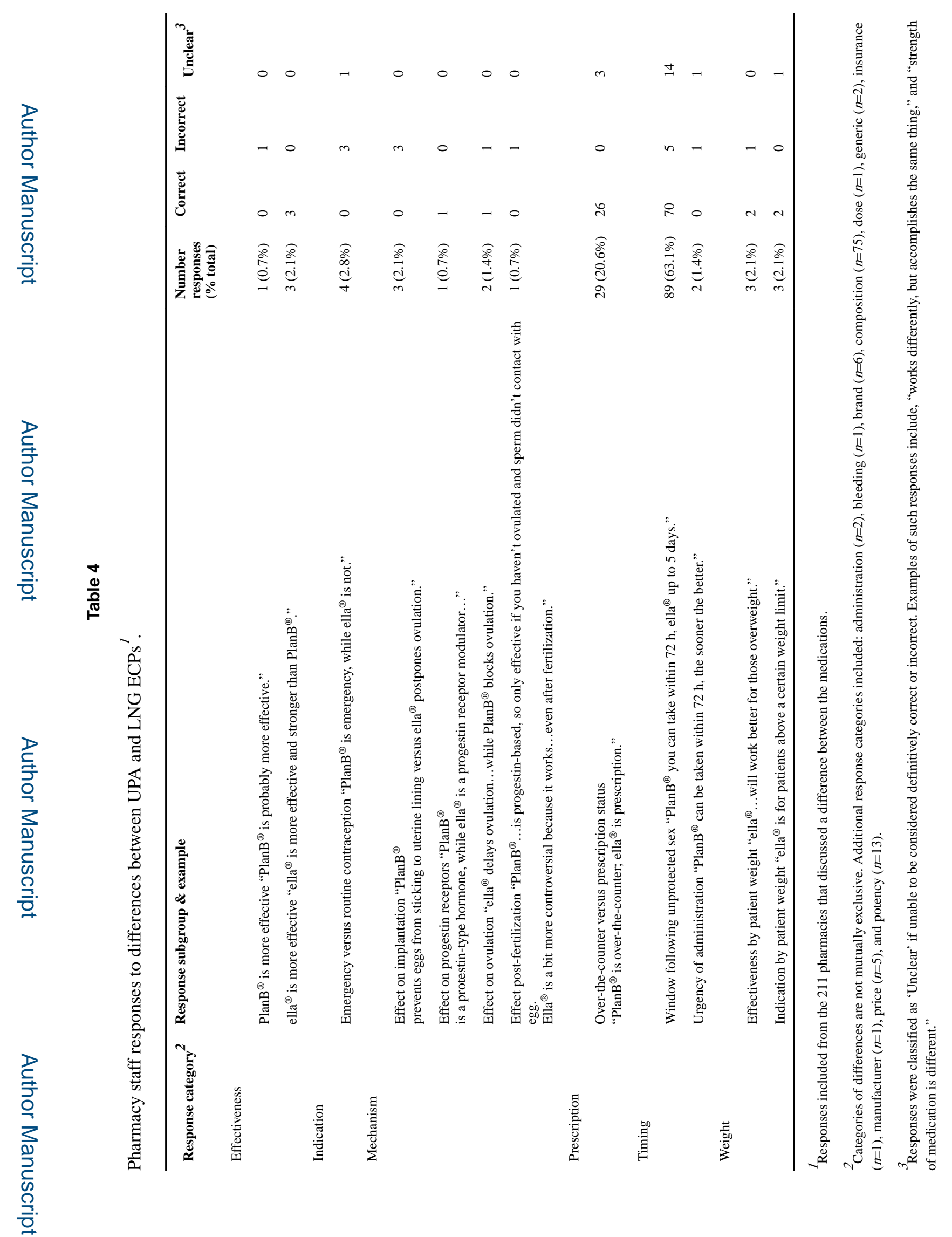

Contraception. Author manuscript; available in PMC 2019 April 16. 